\title{
Polymerization of Sulfur-Containing Aldehydes. III. Copolymerization of Sulfur-Containing Aldehydes with Acetaldehyde and with $n$-Butyraldehyde
}

\author{
Noboru Yамамото and Iwao Yamashita \\ Government Industrial Research Institute, Osaka \\ Midorigaoka 1, Ikeda, Osaka, Japan. \\ (Received January 10, 1972)
}

\begin{abstract}
Copolymerizations of sulfur-containing aldehydes including $\beta$-methylthiopropaldehyde, $\beta$-methylthiobutyraldehyde, and $\beta$-ethylthiobutyraldehyde with acetaldehyde, as well as, with $n$-butyraldehyde, were carried out at $-78^{\circ} \mathrm{C}$ in toluene using $\mathrm{AlEt}_{3}$ as an initiator. From the examination of the solubility, the composition, the infrared spectra, and the partial thermal decomposition of the resultant polymer, it was found that copolymerization with acetaldehyde yielded a random copolymer, while copolymerization with $n$-butyraldehyde produced the copolymer having block units. The facts were also supported by the determination of the apparent values of monomer reactivity ratios. The thermal stability of the copolymers was fairly good in comparison with the homopolymers of conventional higher aliphatic aldehydes.

KEY WORDS Copolymerization / Acetaldehyde / $n$-Butyraldehyde / Triethylaluminum / Thermal Decomposition / Monomer Reactivity Ratio / Sulfur-Containing Aldehyde /
\end{abstract}

Recently, many papers ${ }^{1-7}$ have dealt with copolymerization of acetaldehyde with other aldehydes on the basis of a standpoint of production of polyaldehyde having enough thermal stability for practical use. The preceding paper $^{8}$ described the polymerization of several sulfur-containing aldehydes to polyacetals having remarkably improved thermal stability and solubility. In this connection, the copolymerization of sulfur-containing aldehydes and a conventional higher aliphatic aldehydes including acetaldehyde and $n$-butyraldehyde was investigated.

\section{EXPERIMENTAL}

\section{Materials}

The preparation and purification of sulfurcontaining aldehydes were carried out by the method described in the preceding paper. Acetaldehyde(AA) was prepared by the decomposition of paraldehyde with cupric sulphate, dried with anhydrous magnesium sulphate and rectified. n-Butyraldehyde(BA) was dried with molecular sieves $5 \mathrm{~A}$ and rectified. Commercial $\mathrm{AlEt}_{3}$ was distilled under nitrogen, and used as dilute solution in toluene.

\section{Polymerization Procedure}

Toluene and the monomer mixture were placed in a polymerization tube previously described, cooled to $-20^{\circ} \mathrm{C}$ and catalyst solution added. After five minutes, the mixture was cooled to $-78^{\circ} \mathrm{C}$, degassed and usually kept for $24 \mathrm{hr}$. Polymerization were stopped by the addition of a large amount of methanol. The resulting polymer was separated as the methanol-insoluble precipitate, which was washed with methanol, and dried. In no case polymer was recovered from the methanol-soluble parts. In the experiments the determination of copolymerization parameters in toluene, the polymerization was stopped at conversions of 5-10\%.

\section{Confirmation of Copolymers}

The formation of copolymer was confirmed by examination of solubility and infrared spectra of the product.

\section{Acetylation and Fractionation of Polymers}

A solution of acetic anhydride $(5 \mathrm{~m} l)$ in pyridine $(50 \mathrm{ml})$ was added to the polymer $(0.5$ g) contained in a $100-\mathrm{m} l$ round bottom flask. 
The mixture was kept overnight at room temperature. Refluxed for $2 \mathrm{hr}$, cooled and the mixture was poured into ice water. The precipitate was washed with water and ethanol, dried and then fractionated with tetrahydrofuran (THF). The soluble fractions were reprecipitated with water.

\section{Composition Analysis}

The composition of the copolymers were determined from analysis of sulfur content by combustion technique.

\section{Monomer Reactivity Ratios}

The apparent values of monomer reactivity ratio were determined by the intersection method, neglecting contribution of depolymerization reaction.

\section{RESULTS AND DISCUSSION}

\section{Copolymerization with $A A$}

Copolymerization of $\beta$-ethylthiobutyraldehyde (ETBA) with $A A$. Table I shows the results of copolymerization using $\mathrm{AlEt}_{3}$ as the initiator with various initial feed compositions of the two monomers. The copolymer obtained with monomer feed ratio of $50 / 50$ was a resinous material of slight elasticity which was completely soluble in THF and in chloroform. As is shown in the infrared spectrum of the copolymer in Figure 1, the peaks observed in the spectra of both homopolymers were found, but the absorption peaks of ether group at $900-1150 \mathrm{~cm}^{-1}$ become simpler than those of both homopolymers. Some new peaks are also observed, which are not seen in the spectrum of the mixture of both homopolymers.
The copolymer produced with initial monomer composition ratio $\mathrm{ETBA} / \mathrm{AA}=25 / 75$ contained partly the THF-soluble fraction. However, the infrared spectrum of the insoluble fraction completely coincided with that of the soluble one. The homopolymerization of AA with $\mathrm{AlEt}_{3}$ yield methanol-soluble amorphous polymer, as well as, crystalline polymer insoluble

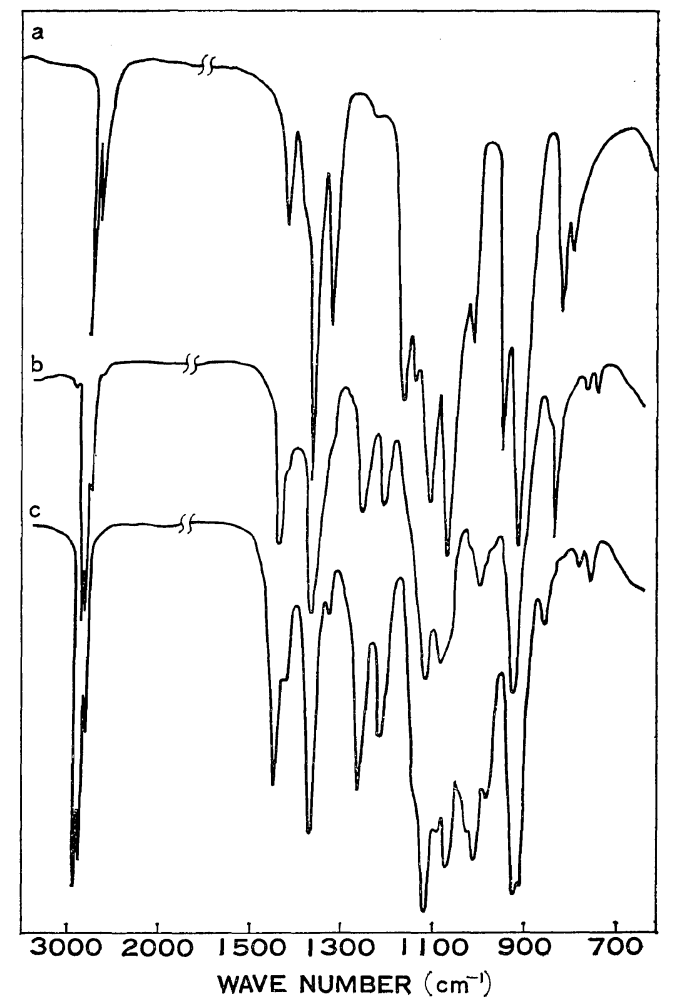

Figure 1. IR spectra of (a) polyAA, (b) copolymer of AA with ETBA, and (c) polyETBA.

Table I. Copolymerization of ETBA with acetaldehyde (AA) ${ }^{\mathrm{a}}$

\begin{tabular}{|c|c|c|c|c|c|c|c|}
\hline \multirow{2}{*}{\multicolumn{2}{|c|}{$\begin{array}{l}\text { Feed monomer, } \\
\text { mol } \%\end{array}$}} & \multicolumn{3}{|c|}{ Copolymer } & \multicolumn{3}{|c|}{ Fractionation } \\
\hline & & \multirow{2}{*}{$\begin{array}{c}\text { Yield, } \\
\%\end{array}$} & \multicolumn{2}{|c|}{ Composition, $\mathrm{mol} \%$} & \multicolumn{2}{|c|}{ THF-soluble } & \multirow{2}{*}{$\begin{array}{c}\text { THF-insoluble, } \\
\%\end{array}$} \\
\hline ETBA & AA & & ETBA & AA & $\%$ & $\eta_{\mathrm{sp}} / c, \mathrm{~d} l / \mathrm{g}^{\mathrm{b}}$ & \\
\hline 75 & 25 & 16 & 63 & 37 & 100 & 14.7 & 0 \\
\hline 50 & 50 & 46 & 45 & 55 & 100 & 7.1 & 0 \\
\hline 25 & 75 & 29 & 21 & 79 & 65 & & 35 \\
\hline 20 & 80 & 10 & 17 & 83 & 44 & & 56 \\
\hline
\end{tabular}

a Total monomer, $0.05 \mathrm{~mol}$; toluene, $12 \mathrm{~m} l$; temp, $-78^{\circ} \mathrm{C}$; time, $24 \mathrm{hr}$; catalyst, $\mathrm{AlEt}_{3}, 0.0005 \mathrm{~mol}$.

b $0.1 \mathrm{~g} / 100 \mathrm{~m} l$ in $\mathrm{THF}$, at $30^{\circ} \mathrm{C}$. 


\section{N. YAmamoto and I. Yamashita}

in THF and in chloroform. Because of the absence of polymer in the methanol-soluble part, the possibility of homopolymerization of AA was eliminated. Attempts at fractional precipitation by using various precipitants such as water, ethanol, methanol, and carbon tetrachloride failed. This fact seems to indicate the absence of ETBA homopolymer. When the copolymerization product prepared with the monomer feed ratio of 50/50 was heated under reduced pressure at $130^{\circ} \mathrm{C}$ for $3 \mathrm{hr}, 22 \%$ of weight loss was observed. The composition and infrared spectra of the copolymerizate measured before and after the thermal decomposition, however, were essentially identical. These observation may suggest the absence of each of both homopolymers/or copolymer having long-chain block units of each component, because of the remarkable difference in the thermal stability of both homopolymers.

Copolymerization of $\beta$-Methylthiobutyraldehyde (MTBA) with AA. Table II shows the results of the copolymerization of MTBA with AA. When the acetaldehyde content in the initial monomer mixture was below $50 \mathrm{~mol} \%$, the copolymerizate were completely soluble in THF. The formation of a random copolymer was confirmed by the same method as those discussed above. In some copolymerization experiments, with AA feed ratios over $80 \%$, a small amount of insoluble polymer was obtained in addition to the THF-soluble copolymer. This insoluble part gave a different infrared spectrum to that of the soluble copolymer, and this observation suggests the formation of copolymer of longer $\mathrm{AA}$ units. When polymerization was stopped at low conversion at all monomer feed ratios, only the soluble copolymer was produced.

Copolymerization of $\beta$-Methylthiopropaldehyde (MTPA) with $A A$. In the homopolymerization of either monomers, the product contained partially THF-insoluble fraction. The copolymer prepared from an equimolar mixture of both monomers was completely soluble in the same solvent. In other monomer feed ratios some insoluble parts were separated, but their infrared spectra were found to be identical with those of the soluble copolymer of similar composition. The copolymers obtained at low conversions were completely soluble in THF at all ratios of feed monomer composition. Therefore, there is no possibility of the occurrence of both homopolymers. The copolymer obtained with monomer ratios of AA to MTPA 50/50 was a white elastic material, and copolymers isolated with other monomer feed ratio were hard resinous materials.

\section{Copolymerization with $B A$}

Copolymerization of ETBA with BA. The results are shown in Table III. Homopolymer of BA obtained using $\mathrm{AlEt}_{3}$ was insoluble in the usual organic solvents. Copolymers obtained with the monomer feed ratio of BA to ETBA $50 / 50$ or $25 / 75$ were completely soluble in THF. PolyETBA was also soluble in this solvent, but the possibility of homopolymer formation was eliminated from the analysis of the infrared spectra before and after thermal decomposition $\left(150^{\circ} \mathrm{C}, 3 \mathrm{hr}\right.$, weight loss $\left.25 \%\right)$ of copolymerizates. The copolymerization products having initial ETBA/BA molar ratios of $25 / 27$ con-

Table II. Copolymerization of MTBA with $\mathrm{AA}^{\mathrm{a}}$

\begin{tabular}{|c|c|c|c|c|c|c|c|}
\hline \multirow{2}{*}{\multicolumn{2}{|c|}{$\begin{array}{l}\text { Feed monomer, } \\
\text { mol } \%\end{array}$}} & \multicolumn{3}{|c|}{ Copolymer } & \multicolumn{3}{|c|}{ Fractionation } \\
\hline & & \multirow{2}{*}{$\begin{array}{c}\text { Yield, } \\
\%\end{array}$} & \multicolumn{2}{|c|}{ Composition, mol $\%$} & \multicolumn{2}{|c|}{ THF-soluble } & \multirow{2}{*}{$\underset{\%}{\text { THF-insoluble }}$} \\
\hline MTBA & AA & & MTBA & AA & $\%$ & $\eta_{\mathrm{sp}} / c, \mathrm{~d} l / \mathrm{g}^{\mathrm{b}}$ & \\
\hline 84 & 16 & 58 & 86 & 14 & 100 & 4.9 & 0 \\
\hline 70 & 30 & 20 & 69 & 31 & 100 & 3.6 & 0 \\
\hline 47 & 53 & 29 & 45 & 55 & 100 & 4.5 & 0 \\
\hline 31 & 69 & 22 & 22 & 78 & 95 & & 5 \\
\hline 16 & 84 & 25 & 9 & 91 & 88 & & 12 \\
\hline
\end{tabular}

a Total monomer, $0.05 \mathrm{~mol}$; catalyst, $\mathrm{AlEt}_{3}, 0.0005 \mathrm{~mol}$; bulk; temp, $-78^{\circ} \mathrm{C}$; time, $24 \mathrm{hr}$.

b $0.1 \mathrm{~g} / 100 \mathrm{~m} l$ in THF, at $30^{\circ} \mathrm{C}$. 
Copolymerization of Sulfur-Containing Aldehydes with Acetaldehyde

Table III. Copolymerization of ETBA with n-BA ${ }^{a}$

\begin{tabular}{|c|c|c|c|c|c|c|c|}
\hline \multirow{2}{*}{\multicolumn{2}{|c|}{$\begin{array}{l}\text { Feed monomer, } \\
\text { mol } \%\end{array}$}} & \multicolumn{3}{|c|}{ Copolymer } & \multicolumn{3}{|c|}{ Fractionation } \\
\hline & & \multirow{2}{*}{$\underset{\%}{\text { Yield, }}$} & \multicolumn{2}{|c|}{ Composition, mol $\%$} & \multicolumn{2}{|c|}{ THF-soluble } & \multirow{2}{*}{$\begin{array}{c}\text { THF-insoluble, } \\
\%\end{array}$} \\
\hline ETBA & BA & & ETBA & $\mathrm{BA}$ & $\%$ & $\eta_{\mathrm{sp}} / c, \mathrm{~d} l / \mathrm{g}^{\mathrm{b}}$ & \\
\hline 75 & 25 & 55 & 69 & 31 & 100 & 17.5 & 0 \\
\hline 50 & 50 & 69 & 38 & 62 & 100 & 7.1 & 0 \\
\hline 25 & 75 & 44 & 27 & 73 & 54 & 5.8 & 46 \\
\hline
\end{tabular}

a Total monomer, $0.05 \mathrm{~mol}$; bulk polymerization; catalyst, $\mathrm{AlEt}_{3}, 0.001 \mathrm{~mol}$; temp, $-78^{\circ} \mathrm{C}$; time, $24 \mathrm{hr}$.

b $0.1 \mathrm{~g} / 100 \mathrm{ml} \mathrm{THF}$, at $30^{\circ} \mathrm{C}$.

tained both the THF-soluble fraction and the insoluble one. However, both fractions gave the same values of elemental analysis. These results seem to suggest the formation of homogeneous copolymers.

Copolymerization of MTBA with BA. When the BA content in the initial monomer mixture was below $50 \mathrm{~mol} \%$, the copolymerizates were throughly soluble in THF unlike the BA-homopolymer. The soluble homopolymer of MTBA was not isolated by fractionation with mixed precipitant such as methanol-ether and ethanol -ether from the copolymerization product. The absence of MTBA-homopolymer was also confirmed by the thermal decomposition method. The polymers having an initial MTBA/BA molar ratio between $40 / 60$ and $10 / 90$ were wholly insoluble in THF, but the composition analysis showed the existence of MTBA units. This indicates the formation of copolymer and the absence of MTBA-homopolymer which was completely soluble in THF. Consequently, the insolubility of the product copolymer seemingly owed to the increase of the length of BA blocks. However, the possibility of coexistence of BAhomopolymer was excluded by the examination of the partial thermal decomposition of the copolymer at temperature which polyBA decompose completely.

Copolymerization of MTPA with BA. All of the polymers produced were sparingly soluble in common organic solvents like the two homopolymers. The infrared spectrum of copolymers was not so different from that of polymer blend. Therefore, it may be assumed that copolymers of long block of both units were produced in all feed monomer ratios. An acetylated sample of the product was fractionated with THF to soluble and insoluble parts. The infrared spectra of both fractions were similar except that the intensity of the absorption at $1460 \mathrm{~cm}^{-1}$ attributed to BA units stronger in the latter case. This observation indicatd that THF-insoluble fraction have more block of BA units, which were also supported by increase of MTPA units after partial thermal decomposition of the product.

\section{Monomer Reactivity Ratio}

The experimental results mentioned above led us to the conclusion that the copolymerizates with AA were random copolymers, while those with BA in all cases, contained fairly long blocks of both units.

In this connection, the copolymerization parameters were tentatively estimated using the Mayo-Lewis equation ${ }^{9}$ and neglecting the effect of depolymerization. The apparent values of monomer reactivity ratio are collected in Table IV. Figure 2 shows examples of a copolymerization curve. In the $\operatorname{MTBA}\left(\mathrm{M}_{1}\right)-\mathrm{AA}\left(\mathrm{M}_{2}\right)$ system, both $r_{1}$ and $r_{2}$ were less than unity, being the product of the values equal to 0.3 . In the $\operatorname{MTBA}\left(\mathrm{M}_{1}\right)-\mathrm{BA}\left(\mathrm{M}_{2}\right)$ system, the product $r_{1} r_{2}$ were more than unity, which suggests the formation of copolymers of considerable block character.

Table IV. Monomer reactivity ratios

\begin{tabular}{ccccl}
\hline $\mathrm{M}_{1}$ & $\mathrm{M}_{2}$ & $r_{1}$ & \multicolumn{1}{c}{$r_{2}$} & $r_{1} \times r_{2}$ \\
\hline MTBA & AA & $0.36 \pm 0.07$ & $0.74 \pm 0.1$ & 0.26 \\
MTPA & BA & $1.15 \pm 0.06$ & $1.39 \pm 0.09$ & 1.6 \\
MTBA & BA & $0.87 \pm 0.08$ & $2.68 \pm 0.18$ & 2.3 \\
\hline
\end{tabular}




\section{N. Yamamoto and I. Yamashita}

Table V. Thermal stability of copolymers ${ }^{a}$

\begin{tabular}{ccc}
\hline \multirow{2}{*}{$\begin{array}{c}\text { Copolymer } \\
\text { composition, mol\% }\end{array}$} & \multicolumn{2}{c}{ Weight loss, \%/hr } \\
\cline { 2 - 3 } & $100^{\circ} \mathrm{C}$ & $130^{\circ} \mathrm{C}$ \\
\hline MTPA/AA $(71 / 29)$ & 1.5 & 7.4 \\
" $(39 / 61)$ & 5.7 & \\
MTBA/AA $(63 / 37)$ & 2.0 & \\
" $\quad(51 / 49)$ & 2.2 & \\
ETBA/AA $(45 / 55)$ & 0.8 & 7.4 \\
MTPA/BA $(44 / 56)^{\mathrm{b}}$ & 1.1 & 7.6 \\
MTBA/BA $(45 / 55)^{\mathrm{b}}$ & 0.3 & 4.0 \\
ETBA/BA $(38 / 69)^{\mathrm{b}}$ & 0.3 & 5.8 \\
AA & 15.0 & 50.0 \\
BA & 6.2 & 30.6
\end{tabular}

a Polymer samples were decomposed by heating for 2-6 hr under the reduced pressure.

b Non-acetylated sample.

\section{Thermal Stability}

The thermal stabilities of the copolymers were compared with those of both homopolymers by measuring the weight loss on heating in air at $100^{\circ} \mathrm{C}$ and at $130^{\circ} \mathrm{C}$. The results are given in Table V. The copolymers were more stable than polyAA and than polyBA, but were less stable than homopolymers of sulfur-containing aldehydes. The copolymers can also be stabilized by the addition of polymeric basic compounds.

Acknowledgement. The authors wish to thank Professor T. Tsuruta of Tokyo university for many helpful discussions.

\section{REFERENCES}

1. H. F. Mark, and N. Ogata, J. Polym. Sci., Part

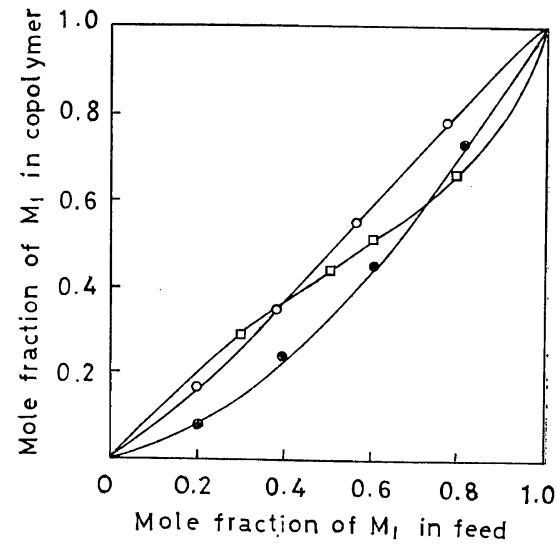

Figure 2. Composition curve for the copolymerization:, MTBA $\left(\mathrm{M}_{1}\right)+\mathrm{BA} ; \bigcirc, \operatorname{MTPA}\left(\mathrm{M}_{1}\right)+$ BA; $\square$, MTBA $\left(\mathrm{M}_{1}\right)+$ AA.

A, 1, 3439 (1963).

2. A. Tanaka, et al., Kobunshi Kagaku (Chem. High Polymers), 20, 687 and 694 (1963); 22, 216, 220, 317, and 321 (1965); A. Tanaka, Y. Hozumi, K. Hatada, and R. Fujishige, J. Polym. Sci., Part B, 2, 181 (1964).

3. T. Iwata, G. Wasai, T. Saegusa, and J. Furukawa, Makromol. Chem., 77, 229 (1964).

4. T. Iwata, T. Saegusa, H. Fujii, and T. Furukawa, ibid., 97, 49 (1966).

5. H. Takida and K. Noro, Kobunshi Kagaku (Chem. High Polymers), 22, 473 (1965).

6. H. Fujii, T. Fujii, T. Saegusa, and J. Furukawa, Makromol. Chem., 63, 147 (1963).

7. H. Takida and K. Noro, Kobunshi Kagaku (Chem. High Polymers), 22, 463 (1965).

8. N. Yamamoto and I. Yamashita, Polymer J., 3, 704 (1972).

9. F. R. Mayo and F. M. Lews, J. Amer. Chem. Soc., 66, 1594 (1944). 